\title{
Arben Merkoçi (Ed.): Paper based sensors
}

\section{Teresa Fernández-Abedul ${ }^{1}$}

Accepted: 4 March 2021 / Published online: 30 March 2021

(C) Springer-Verlag GmbH Germany, part of Springer Nature 2021

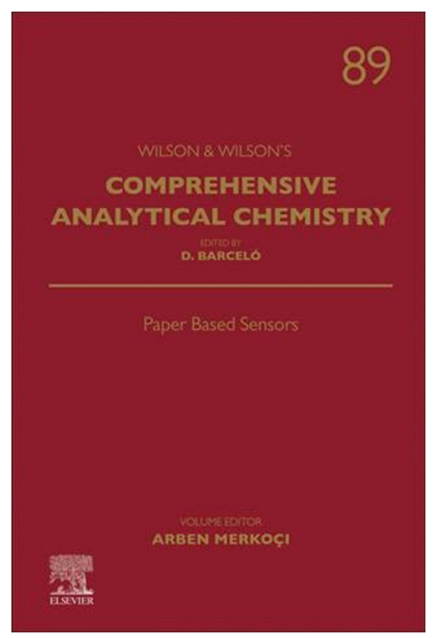

\section{Bibliography}

Paper based sensors

Arben Merko i (Ed.)

Series: Wilson \& Wilson's

Comprehensive Analytical

Chemistry, Vol. 89

ISBN: 978-0-444-64345-2

Elsevier

Hardcover, 478 pages,

11 th June $2020, \$ 255,00 /$

$€ 251.45$

Book's topic The field of sensors is experiencing an enormous and continuous advance, leading most of the rankings of publications in categories related to Analytical Chemistry. Nowadays, the interest on devices that allow decentralization of analysis is huge. The society requires on-site assays not only in the clinical field (as seen in the current pandemic situation) but in many other areas such as agro-food or environmental monitoring, among other. Accordingly, there is an explosion of autonomous, small and fieldable platforms that are being adapted advantageously to different applications. They usually contain low-cost disposable elements and integrate several steps of the analytical process. Here, paper plays an important role, especially for sensor fabrication. Although it is a well-known material in (bio)chemical analysis, it has been reinvented opening new possibilities. In this context, the 89th volume Paper based sensors by A. Merko i, from the Wilson \& Wilson's Comprehensive

M. Teresa Fernández-Abedul mtfernandeza@uniovi.es

1 Departamento de Química Física y Analítica, Universidad de Oviedo, Avda. Julián Clavería, 8, 33006 Oviedo, Asturias, Spain
Analytical Chemistry edited by D. Barceló, is an excellent reference, not only for starters but also for providing insight in a field that is extremely promising. Analysis is moving from the bench to the field in a continuous and fast race, and here, portable and simple devices, providing sensitive and specific methodologies are required. The inclusion of this topic, paperbased sensors, in this prestigious encyclopedia, dedicated to recent developments and interesting applications of Analytical Chemistry, indicates the enormous interest and also the maturity of this field. A special attempt has been made to include all the different aspects involved in sensor development: design, fabrication, detection... based on the point of view and knowledge of experts working on the subject, with many references of current literature.

Contents The book is built on 12 chapters for a total of 478 pages. The first chapter explores paper as a substrate for electrochemical micro-devices, being this principle of detection the most promising in the (bio)sensor field. This is an introductory chapter and, therefore, after commenting the history, types of paper and fabrication procedures (for patterning the paper and also electrodes), applications are included. The second chapter is also an introduction to main bioapplications i.e., detection of biological compounds using enzymatic methods, immunoasays and DNA sensors. Printed-paper based (bio)sensors are considered in the third chapter. Printing allows micropatterning of paper generating small size channels, depositing recognition elements or conducting inks and also enables fast mass production. The following fourth and fifth chapters are devoted to paper-based electrochemical devices, including interesting and new points of view such as the role of paper in electroanalytical devices with special emphasis on its use as reactor as well as on the employ of different electrochemical techniques (amperometric, voltammetric, impedimetric or potentiometric), on the development of lateral and vertical flow paper-based microfluidic devices or on the detection in liquid and gas phases. The number six includes emerging engineering concepts and challenges of paper-based biosensors for clinical and biomedical analysis of common samples (blood and serum, as well as urine). Chapter seven 
is devoted to all the non-enzymatic applications of lab-onpaper platforms, where enzymatic recognition elements are substituted by synthetic ones such as nanostructured materials. Special emphasis is made in chapter eight on lateral flow test strips, dealing with high yield manufacture and including interesting aspects to move platforms from the lab to the market. Understanding the porous materials and their chemistries, the housing of the platforms and yield maximizing as well as total cost determination are considered. The following three chapters (nineth, tenth and eleventh) are more specific, related to nanopaper-based (optical and electrical) sensors, nanoparticlebased lateral flow assays, and flexible cellulose-based devices for monitoring physical parameters. The last chapter (twelfth) presents paper-based sensing platforms as ideal for point-ofcare applications, with interesting focus on non-invasive sampling approaches.

Comparison with the existing literature Analysis with paper-based devices is, undoubtedly, a topic of enormous interest. This is confirmed by the increasing number of scientific articles published in high impact factor journals and in books of international editorials. As a very active editorial, Springer presents three related books: Paper-based diagnostics: current status and future applications (K. J. Land, 2019), Paper microfluidics, theory and applications (S. Bhattacharya, S. Kumar, A.K. Argarwal, 2019, as a part of Advanced functional materials and sensors Series) and Paper-based medical diagnostic devices (J. H. Lee, 2021, as a part of Bioanalysisadvanced materials, methods and devices Series). They include interesting applications, mainly clinical, with emphasis on the developing world, and also different items such as e.g., fluid transport and flow control, or energy storage on paper. This book completes the field filling important gaps, as seen in the already commented contents, being a necessary reference for having an entire view of this mature yet extremely promising area.

Critical assessment The great explosion of paper-based devices has occurred after the appearance of microfluidic paperbased analytical devices ( $\mu$ PADs). They take advantage of the capillary flow (as in first lateral flow assays) but also of the analysis in static sites (as in old spot tests), combining them in original designs. They benefit from properties such as foldability or porosity to generate innovative platforms that incorporate different detection principles. The integration of several steps of the analytical process in the same device has led to the lab-on-a-chip concept. Due to the importance of this material, a specific term has emerged: lab-on-paper. The present book, that covers all these different aspects, is of very useful to have a complete idea of the many possibilities that this outstanding material provides. It will serve as an inspiration for generating even more innovative and useful low-cost platforms, able to perform decentralized analyses in a simple and versatile way.

Readership recommendation The book is addressed to scientists (senior/junior; teachers and $\mathrm{PhD}$ students) working in the development of tools for decentralized analysis. Different principles of detection, mainly electrochemical and optical, are included. Moreover, several steps of the processes performed in a lab could be incorporated with a great versatility in the design. Therefore, promising innovations can motivate scientists of analytical chemistry but also separation science, biochemistry, pharmacy, food safety... apart from those devoted to the clinical or environmental fields, the two main applications. The combination with electronics, mechanics... to generate fieldable instrumentation, paves the way to the collaboration with engineers for developing fully integrated devices, what makes the topic also interesting to these technological areas.

Summary The present volume is an excellent contribution to the field of sensors, in regard to those based on paper. The twelve chapters provide comprehensive state of the art of the field, including reinvented platforms such as those based on lateral flow assays as well as microfluidics. Issues related to the fabrication, the integration of various detection systems and the use of different techniques and innovative approaches as well as the main applications are covered in the volume. As stated in the preface: "The recent COVID-19 pandemics is an evidence of the need of paper-based sensors. Thanks to their cost efficiency, these were between the few devices able to be distributed fast and in big quantities in various parts of the world...Still much work is needed to make paper-based sensors more sensitive and afford even multidetection and address real analytical application scenarios". Reading this book is one of the motivating ways to start with!

\section{Declarations}

Conflict of interest The author declares no competing interests.

Publisher's note Springer Nature remains neutral with regard to jurisdictional claims in published maps and institutional affiliations. 